\title{
Supercritical Fluid Chromatography in Natural Product Analysis - An Update
}

Authors

Nora Gibitz Eisath, Sonja Sturm, Hermann Stuppner

\section{Affiliation}

Institute of Pharmacy, Department of Pharmacognosy, CCB Centrum of Chemistry and Biomedicine, University of Inns-

bruck, Innsbruck, Austria

Key words

supercritical fluid chromatography, SFC, SFC-MS, mass spectrometry, natural product analysis, medicinal plants

received June 16,2017

revised August 24, 2017

accepted August 31, 2017

Bibliography

DOI https://doi.org/10.1055/s-0037-1599461

Published online September 22, 2017 | Planta Med 2018; 84: 361-371 @ Georg Thieme Verlag KG Stuttgart · New York I ISSN 0032-0943

\section{Correspondence}

Univ. Prof. Dr. Hermann Stuppner

Institute of Pharmacy, CCB - Centrum of Chemistry and

Biomedicine, University of Innsbruck, Department of

Pharmacognosy

Innrain 80/82, 6020 Innsbruck, Austria

Phone: + 4351250758400 , Fax: + 4351250758499

hermann.stuppner@uibk.ac.at

\section{ABSTRACT}

The wide chemical diversity of natural products has challenged analysts all over the world and has been a driving force for the development of innovative technologies since decades. In the last years, supercritical fluid chromatography (SFC) has finally emerged from the shadow of liquid chromatography (LC) and gas chromatography (GC) and has become a powerful tool in modern natural product analysis. Whereas in the past the technique had mainly been restricted to a small group of nonpolar compounds, it has largely expanded its suitability in the last years and has demonstrated possibilities without boundaries. This mini-review, focused on the latest applications, provides a brief update on the current status of SFC in natural product analysis with the aim to demonstrate its applicability for both polar and nonpolar plant constituents. The approaches cover the whole range of polarity, including carotenoids, flavonoids, water-unstable ginkgolides, and even highly polar triterpene saponins with several sugar residues.

$\begin{array}{ll}\text { ABBREVIATIONS } \\ \text { APCI } & \text { atmospheric pressure chemical ionization } \\ \text { BPR } & \text { backpressure regulator } \\ \text { DAD } & \text { diode-array detector } \\ \text { DEA } & \text { diethylamine } \\ \text { ESI } & \text { electrospray ionization } \\ \text { LOQ } & \text { limit of quantitation } \\ \text { RP-HPLC } & \text { reversed-phase HPLC } \\ \text { SFC } & \text { supercritical fluid chromatography } \\ \text { SFE } & \text { supercritical fluid extraction } \\ \text { UHPLC } & \text { ultra-high performance liquid chromatography } \\ \text { UHPSFC } & \text { ultra-high performance supercritical } \\ & \text { chromatography }\end{array}$

\section{Introduction}

Already developed more than 50 years ago, SFC was overshadowed by LC and GC for a long time [1]. It was mainly criticized for its weak UV sensitivity, poor quantitative performance, and limited reliability. In the last decade, the introduction of a new generation of commercially manufactured instruments tackled most of these limitations, and SFC has developed to a powerful analytical tool combining advantages of short analysis times and unique selectivity with low operating costs and environmental friendliness $[2,3]$.

The wide chemical diversity of natural products has always challenged analysts. Therefore, the availability of such highly efficient analytical technologies is of tremendous interest [4]. Initially, the application field of SFC in natural product analysis was relatively modest, focusing mainly on nonpolar compounds $[5,6]$. However, the technique has largely expanded its suitability and has become an accepted analytical alternative. As shown in 


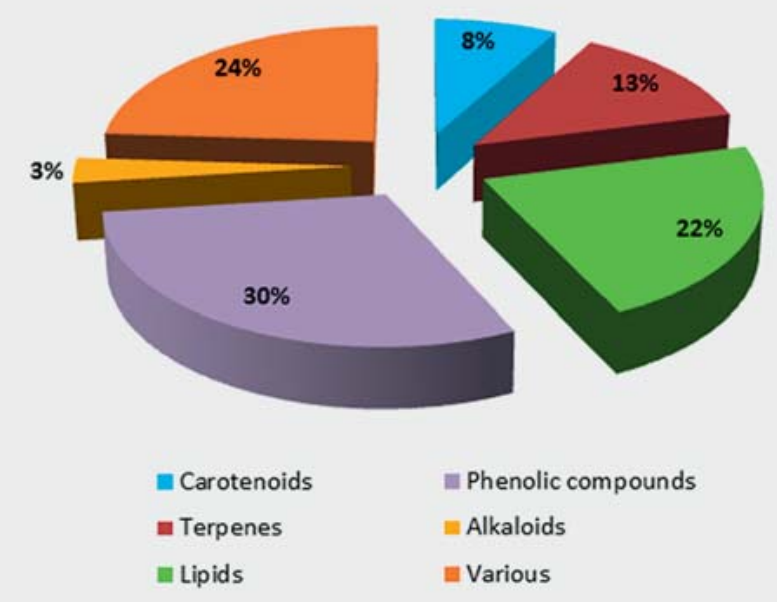

- Fig. 1 Range of SFC applications in natural product analysis categorized by substance classes from 2012 to 2016. Scifinder. Date of information gathering: May 2017.

- Fig. 1, the range of applications is broadly diversified and extends from the separation of nonpolar lipids to the analysis of highly polar triterpene saponins with several sugar residues $[3,4$, $7,8]$.

The number of publications dealing with the application of SFC for natural product analysis is increasing steadily ( $\bullet$ Fig. 2), demonstrating its potential as complementary alternative to other well-established techniques as (U)HPLC or GC. These remarkable advances since the last review [7], enlightening the role of SFC in plant analysis, motivated us to present a brief update focusing on applications of the last two years.

\section{Theoretical Background and Instrumentation}

SFC operation is based on the use of a supercritical fluid as mobile phase. The supercritical condition is obtained whenever pressure and temperature of a gas or a liquid exceed their critical values [9]. In this state, features of both, the liquid and the gaseous state, are connected in a unique way: high dissolving capabilities and densities like a liquid are paired with low viscosity and high diffusivity of a gas $[10,11]$.

Nowadays, supercritical $\mathrm{CO}_{2}$ is most widely used, because its critical values $\left(31^{\circ} \mathrm{C}\right.$ and 74 bar) are easily attainable and it is inert, nontoxic, readily available, and cheap. Additionally, it is also an environmental friendly alternative to the standard organic solvents $[10,12]$. Supercritical $\mathrm{CO}_{2}$ is a highly lipophilic solvent with a polarity similar to hydrocarbons [13]. Therefore, SFC is often incorrectly considered as a normal phase system [14]. Analysis of more polar solutes requires the addition of an organic modifier (primarily an alcohol) $[15,16]$. This modification causes an increase of the critical point with the consequence that most separations do not occur under supercritical but rather under so-called subcritical conditions. Due to the fact that both states have comparable characteristics, it is not of particular importance for the operator $[17,18]$.

The introduction of modern state-of-the art instruments (also called UHPSFC) by several manufacturers was the major driving force for the renewed interest in SFC in recent years [2]. Although these innovative systems are largely based on UHPLC technology, the use of a supercritical fluid as mobile phase requires several important setup adaptations [18]: a BPR is required to enable accurate control of the pressure and an adapted pumping system to fulfil the mobile phase characteristics [13]. Modern instruments benefit from an optimized BPR device that not only reduces pressure variations during analysis but also ensures quicker adaptations to changes in flow rate and mobile phase composition [19]. Revised $\mathrm{CO}_{2}$ delivery systems, guaranteeing adequate cooling of the incoming $\mathrm{CO}_{2}$ to insure its liquid state, are another key factor for the new, reliable SFC performance $[13,18]$. In addition, the new instruments include lower injection volumes, reduced void volumes to limit band broadening, and higher upper pressure limits $[2,19]$. Although the pressure limits (400 to 600 bar) are still quite low compared to UHPLC systems (over 1000 bar), this is mostly not a limiting factor due to the fact that the much lower viscosity of $\mathrm{CO}_{2}$-based mobile phases generates only low pressure drops compared to liquids used in UHPLC [13]. Nearly all stationary phases and column designs tailored for HPLC, including columns packed with sub-2- $\mu \mathrm{m}$ and core-shell particles, are suitable for SFC as well [19-23]. Recently, the ongoing interest in SFC as a potent analytical technique led to an increasing availability of stationary phases specifically designed for SFC use [22]. Modern SFC systems are compatible with a wide range of different detectors, including MS, evaporative light scattering detector, and DAD [13, 24]. The latter was often criticized for its low sensitivity, largely attributed to density and refraction index changes, caused by pressure oscillations. This pressure-induced UV noise was another factor that could be significantly improved by the introduction of the modernized BPR devices $[19,25]$. Beside DAD detection, the hyphenation of SFC to MS is continuously growing in importance. While in the past APCI was considered as prevailing ionization source, ESI gained in popularity in the last years [26].

Among these technical improvements also the recent introduction of a fully automated system, combining online SFE and SFC with MS detection in a single flow path, is of great interest for natural product analysis. The addition of polar organic solvents to the supercritical $\mathrm{CO}_{2}$ allows the extraction and simultaneous analysis of compounds with a wide range of polarities and makes it to an interesting future approach $[27,28]$.

More information about the theoretical background, instrumentation, practical approaches, and different applications are available in recent publications [6, 9, 13, 18, 22, 29-32].

\section{Applications on Natural Products}

For a long time, SFC analysis focused on nonpolar plant ingredients like lipids and carotenoids. In the last years, an increasing interest in the often underestimated potential of SFC for analysis of polar compounds could be observed. The following section, categorized by substance classes, gives an overview of recent SFC applications on natural product analysis with the aim to dem- 


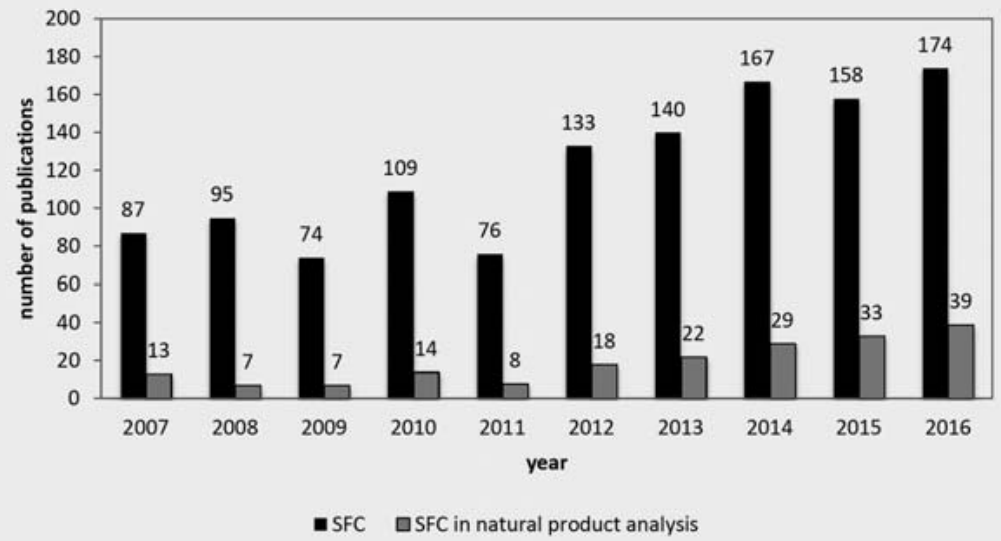

- Fig. 2 Number of publications per year in the field of SFC in general and of SFC in natural product analysis over the last 10 years. Source: Scifinder. Date of information gathering: May 2017.

onstrate its wide applicability for both polar and nonpolar plant constituents. Some selected applications including detailed conditions are listed in $>$ Table $\mathbf{1}$.

\section{Lipids}

Since its introduction, SFC has consequently conquered lipid analysis as one of its major application fields. A vast number of studies focused on the qualitative or quantitative determination of those important food ingredients. Some recent reviews provide an excellent overview of SFC analysis in this field, from the beginnings to the current state including up-to-date applications; recent advances in lipid analysis will therefore not be further discussed [3, 33-37].

\section{Carotenoids}

The use of SFC for the separation of carotenoids was already mentioned in 1968 [38]. Since then, a large number of papers was published, which emphasizes the important role of SFC as an alternative analysis technique in this sector [36]. There are different forms of carotenoids: free carotenoids and more stable forms, esterified with fatty acids [39]. To release all esters and to simplify the analysis, most of the investigations were performed after a saponification step [40]. Two recent applications are worth mentioning as this simplification was avoided to prevent artifact formation and to preserve all information on the natural carotenoid profile.

Bonaccorsi et al. [39] identified more than 100 different compounds belonging to chlorophylls, free carotenes, free xanthophylls, and xanthophyll mono- and diesters in sweet bell peppers through the offline coupling of SFC and LC. The first dimension was performed on a SFC system using an Acquity UPC ${ }^{2}$ HSS $_{18}$ SB column and ethanol as modifier, while the second dimension consisted of a RP-HPLC combined with DAD and MS detection and was performed on a C30 stationary phase. The high orthogonality of SFC and HPLC clearly enhanced the separation power and facilitated the rapid quantitation as well as stability studies of carotenoids in overripe yellow and red bell peppers.
Cleavage of a fragment from the usual carotenoid structure leads to the formation of apocarotenoids. Giuffrida et al. [41] developed the first SFC/MS method for the determination of native apocarotenoids in red habanero pepper, the hottest pepper in the world. Twenty-five apocarotenoids (14 free apocarotenoids and 11 apocarotenoids fatty acid esters) were separated on a novel fused-core C30 column with methanol as modifier in less than $5 \mathrm{~min}$. The compounds were detected by selective ion monitoring in the negative mode utilizing a triple quadrupole mass spectrometer and an APCI interface. Identity was further confirmed by selective reaction monitoring in positive and negative ionization mode.

\section{Terpenes}

Terpenes are a wide-spread group of plant constituents of large chemical diversity. While early investigations were mainly focused on lipophilic terpenes, lately interest in more polar compounds has increased. Particularly noteworthy in this context is a publication by Huang et al. demonstrating the potential of SFC/MS for the analysis of triterpene saponins [42]. Nine kudinosides, six stauntosides, or 11 ginsenosides could be well resolved on a ZORBAX RX-SIL column within 10 min with slightly different parameters. As shown in $\mathbf{F i g . 3}$, addition of $5 \%$ or $10 \%$ water to methanol as modifier was mandatory in all cases to improve resolution and reduce analysis time; $0.05 \%$ formic acid was added to enhance ionization. The methods were successfully applied to the analysis of kudinosides in Ilex latifolia, and ginsenosides in Panax quinquefolius and Panax ginseng.

Twenty years after the first SFC study on Ginkgo biloba, interest has recently been rekindled [43]. Ginkgolic acids and terpene lactones were determined in extracts and dietary supplements using an Acquity UPC ${ }^{2}$ BEH 2-EP column and a mixture of isopropanol/ methanol $(50: 50 \mathrm{v} / \mathrm{v})$ with $10 \mathrm{mmol}$ ammonium acetate as modifier [44]. Quantitation of low concentrations of both ginkgolic acid (LOQs $<100 \mathrm{ng} / \mathrm{mL}$ ) and terpene lactones (LOQs $<1 \mu \mathrm{g} / \mathrm{mL}$ ) could be achieved by single quadrupole MS detection. The developed method might be an alternative to existing methods with 
- Table 1 Selected SFC applications in the analysis of natural products, categorized by substance classes.

\begin{tabular}{|c|c|c|c|c|c|c|}
\hline Compounds & Plant species & Stationary phase & Analytical conditions & Detection & Quant. & Ref. \\
\hline \multicolumn{7}{|l|}{ Carotenoids } \\
\hline Apocarotenoids & $\begin{array}{l}\text { Red habanero } \\
\text { pepper }\end{array}$ & $\begin{array}{l}\text { Ascentis Express C30 } \\
(4.6 \times 150 \mathrm{~mm} \text {, } \\
2.7 \mu \mathrm{m})\end{array}$ & $\begin{array}{l}\mathrm{A}: \mathrm{CO}_{2}, \mathrm{~B}: \mathrm{MeOH} \\
\text { Gradient elution mode, } 2 \mathrm{~mL} / \\
\text { min, } 35^{\circ} \mathrm{C}, 150 \mathrm{bar}\end{array}$ & $\mathrm{MS}(\mathrm{APCl})$ & $\checkmark$ & {$[41]$} \\
\hline Carotenoids & $\begin{array}{l}\text { Chlorella sp. } \\
\text { Scenedesmus sp. } \\
\text { Rosehip }\end{array}$ & $\begin{array}{l}\text { Torus } 1-\mathrm{AA} \\
(3.0 \times 100 \mathrm{~mm} \text {, } \\
1.7 \mu \mathrm{m})\end{array}$ & $\begin{array}{l}\mathrm{A}: \mathrm{CO}_{2}, \mathrm{~B}: \mathrm{MeOH} \\
\text { Gradient elution mode, } 2 \mathrm{~mL} / \\
\text { min, } 35^{\circ} \mathrm{C}, 160 \mathrm{bar}\end{array}$ & $\begin{array}{l}\text { DAD/MS } \\
\text { (ESI) }\end{array}$ & $\checkmark$ & [69] \\
\hline Carotenoids & $\begin{array}{l}\text { Dietary supple- } \\
\text { ments }\end{array}$ & $\begin{array}{l}\text { Acquity UPC }{ }^{2} \text { HSS } \\
\text { C18 SB }(3.0 \times \\
150 \mathrm{~mm}, 1.8 \mu \mathrm{m})\end{array}$ & $\begin{array}{l}\mathrm{A}: \mathrm{CO}_{2}, \mathrm{~B}: \mathrm{MeOH} / \mathrm{EtOH}(1: 2 \mathrm{v} / \mathrm{v}) \\
\text { Gradient elution mode, } 1.8 \mathrm{~mL} / \\
\text { min, } 35^{\circ} \mathrm{C}, 152 \text { bar }\end{array}$ & $\mathrm{DAD}$ & $\checkmark$ & {$[70]$} \\
\hline \multicolumn{7}{|l|}{ Terpenes } \\
\hline $\begin{array}{l}\text { Terpene lactone } \\
\text { and ginkgolic } \\
\text { acids }\end{array}$ & Gingko biloba & $\begin{array}{l}\text { Acquity UPC }{ }^{2} \text { BEH } \\
2-E P(3.0 \times 150 \mathrm{~mm} \text {, } \\
1.7 \mu \mathrm{m})\end{array}$ & $\begin{array}{l}\mathrm{A}: \mathrm{CO}_{2}, \mathrm{~B}: 10 \mathrm{mmol} \text { ammo- } \\
\text { nium acetate in } \mathrm{MeOH} / \mathrm{lsopro}- \\
\text { panol }(1: 1 \mathrm{v} / \mathrm{v}) \\
\text { Gradient elution mode, } 1.4 \mathrm{~mL} / \\
\text { min, } 30^{\circ} \mathrm{C}, 103 \mathrm{bar}\end{array}$ & $\begin{array}{l}\text { DAD/MS } \\
\text { (ESI) }\end{array}$ & $\checkmark$ & {$[44]$} \\
\hline Ginkgolides & Gingko biloba & $(n / a)$ & $\begin{array}{l}\mathrm{A}: \mathrm{CO}_{2}, \mathrm{~B}: 5 \% \text { water and } \\
10 \mathrm{mmol} \text { ammonium acetate } \\
\text { in } \mathrm{MeOH} \\
\text { Gradient elution mode, } 2 \mathrm{~mL} / \\
\text { min, } 40^{\circ} \mathrm{C}, 200 \text { bar }\end{array}$ & MS (ESI) & $\checkmark$ & {$[68]$} \\
\hline $\begin{array}{l}\text { Kudinosides, } \\
\text { stauntosides, and } \\
\text { ginsenosides }\end{array}$ & $\begin{array}{l}\text { Ilex latifolia, Panax } \\
\text { quinquefolius and } \\
\text { Panax ginseng }\end{array}$ & $\begin{array}{l}\text { ZORBAX RX-SIL } \\
(4.6 \times 150 \mathrm{~mm} \text {, } \\
5 \mu \mathrm{m})\end{array}$ & $\begin{array}{l}\mathrm{A}: \mathrm{CO}_{2}, \mathrm{~B}: 5-10 \% \text { water and } \\
0.05 \% \text { formic acid in } \mathrm{MeOH} \\
\text { Gradient elution mode, } 3 \mathrm{~mL} / \\
\text { min, } 20^{\circ} \mathrm{C}, 160 \text { bar }\end{array}$ & $\begin{array}{l}\text { DAD/MS } \\
\text { (ESI) }\end{array}$ & - & {$[42]$} \\
\hline $\begin{array}{l}\text { Ginsenoside, } \\
\text { nucleoside, and } \\
\text { nucleobases }\end{array}$ & Panax ginseng & $\begin{array}{l}\text { ZORBAX RX-SIL } \\
(4.6 \times 150 \mathrm{~mm} \text {, } \\
5 \mu \mathrm{m})\end{array}$ & $\begin{array}{l}\mathrm{A}: \mathrm{CO}_{2}, \mathrm{~B}: 5 \mathrm{mmol} \text { ammonium } \\
\text { acetate in } \mathrm{MeOH} \\
\text { Gradient elution mode, } 3 \mathrm{~mL} / \\
\text { min, } 35^{\circ} \mathrm{C}, 160 \text { bar }\end{array}$ & $\begin{array}{l}\text { DAD/MS } \\
\text { (ESI) }\end{array}$ & - & {$[71]$} \\
\hline $\begin{array}{l}\text { Diterpenoid acids } \\
\text { (continentalic } \\
\text { acid and kaure- } \\
\text { noic acid) }\end{array}$ & Aralia continentalis & $\begin{array}{l}\text { Acquity UPC } 2 \text { Torus } \\
1-\mathrm{AA}(2.1 \times 150 \mathrm{~mm} \text {, } \\
1.7 \mu \mathrm{m})\end{array}$ & $\begin{array}{l}\mathrm{A}: \mathrm{CO}_{2}, \mathrm{~B}: 0.1 \% \text { formic acid in } \\
\mathrm{MeOH} \\
\text { Isocratic elution mode, } 0.6 \mathrm{~mL} / \\
\text { min, } 40^{\circ} \mathrm{C}, 138 \text { bar }\end{array}$ & $\mathrm{DAD}$ & $\checkmark$ & {$[72]$} \\
\hline $\begin{array}{l}\text { Sesquiterpenes } \\
\text { and other con- } \\
\text { stituents }\end{array}$ & $\begin{array}{l}\text { Matricaria chamo- } \\
\text { milla, Chamaeme- } \\
\text { lum nobile }\end{array}$ & $\begin{array}{l}\text { Acquity UPC }{ }^{2} \text { BEH } \\
2-E P(3.0 \times 150 \mathrm{~mm} \text {, } \\
1.7 \mu \mathrm{m})\end{array}$ & $\begin{array}{l}\mathrm{A}: \mathrm{CO}_{2}, \mathrm{~B}: 0.5 \% \text { formic acid in } \\
\mathrm{MeOH} / \text { Isopropanol }(1: 1 \mathrm{v} / \mathrm{v}) \\
\text { Gradient elution mode, } 1.7 \mathrm{~mL} / \\
\text { min, } 50^{\circ} \mathrm{C}, 103 \text { bar }\end{array}$ & $\begin{array}{l}\text { DAD/MS } \\
\text { (ESI) }\end{array}$ & - & {$[73]$} \\
\hline Camphor & $\begin{array}{l}\text { Tanacetum } \\
\text { parthenium }\end{array}$ & $\begin{array}{l}\text { Acquity UPC }{ }^{2} \text { BEH } \\
2-E P(3.0 \times 100 \mathrm{~mm} \text {, } \\
1.7 \mu \mathrm{m})\end{array}$ & $\begin{array}{l}\mathrm{A}: \mathrm{CO}_{2}, \mathrm{~B} \text { : Isopropanol } \\
\text { Gradient elution mode, } 2.0 \mathrm{~mL} / \\
\text { min, } 50^{\circ} \mathrm{C}, 138 \mathrm{bar}\end{array}$ & $\mathrm{DAD}$ & $\checkmark$ & {$[74]$} \\
\hline \multicolumn{7}{|l|}{ Alkaloids } \\
\hline $\begin{array}{l}\text { Indole and oxin- } \\
\text { dole alkaloids }\end{array}$ & $\begin{array}{l}\text { Mitragyna speci- } \\
\text { cosa }\end{array}$ & $\begin{array}{l}\text { Agilent RX-SIL } \\
(2.1 \times 50 \mathrm{~mm} \text {, } \\
1.8 \mu \mathrm{m})\end{array}$ & $\begin{array}{l}\mathrm{A}: \mathrm{CO}_{2}, \mathrm{~B}: 10 \mathrm{mmol} \text { ammonium } \\
\text { acetate in } \mathrm{MeOH} \\
\text { Gradient elution mode, } 0.5 \mathrm{~mL} / \\
\text { min, } 25^{\circ} \mathrm{C}, 180 \text { bar }\end{array}$ & $\mathrm{DAD}$ & - & {$[46]$} \\
\hline \multirow[t]{2}{*}{$\begin{array}{l}\text { Spiro oxindole } \\
\text { alkaloids }\end{array}$} & $\begin{array}{l}\text { Uncaria macro- } \\
\text { phylla }\end{array}$ & $\begin{array}{l}\text { Torus } 1-\mathrm{AA} \\
(3.0 \times 100 \mathrm{~mm} \text {, } \\
1.7 \mu \mathrm{m})\end{array}$ & $\begin{array}{l}\mathrm{A}: \mathrm{CO}_{2}, \mathrm{~B}: 0.1 \% \mathrm{DEA} \text { in } \mathrm{ACN} \\
\text { Isocratic elution mode, } 1.2 \mathrm{~mL} / \\
\text { min, } 45^{\circ} \mathrm{C}, 138 \text { bar }\end{array}$ & $\mathrm{DAD}$ & - & [48] \\
\hline & & $\begin{array}{l}\text { Torus Diol } \\
(3.0 \times 100 \mathrm{~mm} \text {, } \\
1.7 \mu \mathrm{m})\end{array}$ & $\begin{array}{l}\mathrm{A}: \mathrm{CO}_{2}, \mathrm{~B}: 0.1 \% \text { ammonium } \\
\text { hydroxide in } \mathrm{ACN} \\
\text { Isocratic elution mode, } 1.2 \mathrm{~mL} / \\
\text { min, } 30^{\circ} \mathrm{C}, 124 \text { bar }\end{array}$ & & & \\
\hline $\begin{array}{l}\text { Aconitum alka- } \\
\text { loids }\end{array}$ & $\begin{array}{l}\text { Aconitum pendu- } \\
\text { lum }\end{array}$ & $\begin{array}{l}\text { Acquity UPC }{ }^{2} \text { BEH } \\
2-E P(2.1 \times 150 \mathrm{~mm} \text {, } \\
1.7 \mu \mathrm{m})\end{array}$ & $\begin{array}{l}\mathrm{A}: \mathrm{CO}_{2}, \mathrm{~B}: 10 \mathrm{mmol} \text { ammonium } \\
\text { acetate in } \mathrm{MeOH} \\
\text { Gradient elution mode, } 0.8 \mathrm{~mL} / \\
\text { min, } 55^{\circ} \mathrm{C}, 145 \text { bar }\end{array}$ & $\begin{array}{l}\text { DAD/MS } \\
\text { (ESI) }\end{array}$ & $\checkmark$ & $\begin{array}{l}{[49]} \\
\text { contin- } \\
\text { ued }\end{array}$ \\
\hline
\end{tabular}


- Table 1 Continued

\begin{tabular}{|c|c|c|c|c|c|c|}
\hline Compounds & Plant species & Stationary phase & Analytical conditions & Detection & Quant. & Ref. \\
\hline $\begin{array}{l}\text { Sesquiterpene } \\
\text { pyridine alkaloids }\end{array}$ & $\begin{array}{l}\text { Tripterygium } \\
\text { wilfordii }\end{array}$ & $\begin{array}{l}\text { Acquity UPC }{ }^{2} \text { BEH } \\
2-E P(2.1 \times 50 \mathrm{~mm} \text {, } \\
1.7 \mu \mathrm{m})\end{array}$ & $\begin{array}{l}\mathrm{A}: \mathrm{CO}_{2}, \mathrm{~B}: \mathrm{MeOH} \\
\text { Gradient elution mode, } 1 \mathrm{~mL} / \\
\min , 45^{\circ} \mathrm{C}, 138 \text { bar }\end{array}$ & $\begin{array}{l}\mathrm{DAD} / \mathrm{MS} \\
\text { (ESI) }\end{array}$ & - & [75] \\
\hline Indole alkaloids & Alstonia scholaris & $\begin{array}{l}\text { Acquity } \mathrm{UPC}^{2} \mathrm{BEH} \\
2-\mathrm{EP}(3.0 \times 100 \mathrm{~mm} \text {, } \\
1.7 \mu \mathrm{m})\end{array}$ & $\begin{array}{l}\mathrm{A}: \mathrm{CO}_{2}, \mathrm{~B}: 2 \mathrm{mmol} \text { ammonium } \\
\text { formate in } \mathrm{MeOH} \\
\text { Gradient elution mode, } 1.5 \mathrm{~mL} / \\
\text { min, } 50^{\circ} \mathrm{C}, 138 \mathrm{bar}\end{array}$ & MS (ESI) & $\checkmark$ & [67] \\
\hline Indole alkaloids & Evodiae frucuts & $\begin{array}{l}\text { Acquity } \mathrm{UPC}^{2} \mathrm{BEH} \\
(3.0 \times 100 \mathrm{~mm} \\
1.7 \mu \mathrm{m})\end{array}$ & $\begin{array}{l}\mathrm{A}: \mathrm{CO}_{2}, \mathrm{~B}: \mathrm{MeOH} \\
\text { Gradient elution mode, } 2 \mathrm{~mL} / \\
\text { min, } 35^{\circ} \mathrm{C}, 207 \text { bar }\end{array}$ & DAD & - & [76] \\
\hline \multicolumn{7}{|c|}{ Phenolic compounds } \\
\hline Kavalactons & $\begin{array}{l}\text { Piper methysticum } \\
\text { (Kava-Kava) }\end{array}$ & $\begin{array}{l}\text { Acquity } \mathrm{UPC}^{2} \mathrm{BEH} \\
(3.0 \times 100 \mathrm{~mm} \\
1.7 \mu \mathrm{m})\end{array}$ & $\begin{array}{l}\mathrm{A}: \mathrm{CO}_{2}, \mathrm{~B}: 0.6 \% \mathrm{DEA} \text { in } \mathrm{MeOH} \\
\text { Gradient elution mode, } 1 \mathrm{~mL} / \\
\min , 70^{\circ} \mathrm{C}, 130 \text { bar }\end{array}$ & DAD & $\checkmark$ & {$[56]$} \\
\hline Anthraquinones & Rhubarb & $\begin{array}{l}\text { Acquity } \mathrm{UPC}^{2} \mathrm{HSSC}_{18} \\
\mathrm{SB}(3.0 \times 100 \mathrm{~mm}, \\
1.8 \mu \mathrm{m})\end{array}$ & $\begin{array}{l}\mathrm{A}: \mathrm{CO}_{2}, \mathrm{~B}: 0.05 \% \mathrm{DEA} \text { in } \mathrm{MeOH} \\
\text { Gradient elution mode, } 2 \mathrm{~mL} / \\
\text { min, } 30^{\circ} \mathrm{C}, 150 \text { bar }\end{array}$ & DAD & $\checkmark$ & [57] \\
\hline Coumarins & Angelica dahurica & $\begin{array}{l}\text { Acquity } \mathrm{UPC}^{2} \text { CSH } \\
\text { Fluoro-Phenyl } \\
(3.0 \times 100 \mathrm{~mm} \text {, } \\
1.7 \mu \mathrm{m})\end{array}$ & $\begin{array}{l}\mathrm{A}: \mathrm{CO}_{2}, \mathrm{~B}: 0.1 \% \mathrm{DEA} \text { in } \mathrm{MeOH} \\
\text { Gradient elution mode, } 1.5 \mathrm{~mL} / \\
\text { min, } 30^{\circ} \mathrm{C}, 130 \text { bar }\end{array}$ & DAD & $\checkmark$ & [53] \\
\hline Coumarins & $\begin{array}{l}\text { Ammi visnaga } \\
\text { fruit }\end{array}$ & $\begin{array}{l}\text { Acquity } \mathrm{UPC}^{2} \mathrm{HSSC}_{18} \\
\mathrm{SB}(3.0 \times 100 \mathrm{~mm}, \\
1.8 \mu \mathrm{m})\end{array}$ & $\begin{array}{l}\text { A: } \mathrm{CO}_{2}, \mathrm{~B}: 0.1 \% \text { DEA in } \mathrm{MeOH} / \\
\text { ACN }(1: 1 \mathrm{v} / \mathrm{v}) \\
\text { Gradient elution mode, } 1.5 \mathrm{~mL} / \\
\text { min, } 30^{\circ} \mathrm{C}, 140 \text { bar }\end{array}$ & DAD & $\checkmark$ & [55] \\
\hline Pyranocumarins & $\begin{array}{l}\text { Angelica gigas } \\
\text { Nakai }\end{array}$ & $\begin{array}{l}\text { Acquity UPC }{ }^{2} \text { CSH } \\
\text { Fluoro-Phenyl }(2.1 \times \\
150 \mathrm{~mm}, 1.7 \mu \mathrm{m})\end{array}$ & $\begin{array}{l}\mathrm{A}: \mathrm{CO}_{2}, \mathrm{~B}: \mathrm{EtOH} \\
\text { Isocratic elution mode, } 0.6 \mathrm{~mL} / \\
\text { min, } 35^{\circ} \mathrm{C}, 138 \text { bar }\end{array}$ & DAD & $\checkmark$ & [54] \\
\hline Flavonoids & $\begin{array}{l}\text { Chrysanthemum } \\
\text { marifolium }\end{array}$ & $\begin{array}{l}\text { ZORBAX RX-SIL } \\
(4.6 \times 150 \mathrm{~mm} \\
5 \mu \mathrm{m})\end{array}$ & $\begin{array}{l}\mathrm{A}: \mathrm{CO}_{2}, \mathrm{~B}: 0.1 \% \text { phosphoric acid } \\
\text { in } \mathrm{MeOH} \\
\text { Gradient elution mode, } 3 \mathrm{~mL} \\
\text { min, } 40^{\circ} \mathrm{C}, 200 \text { bar }\end{array}$ & DAD & $\checkmark$ & [51] \\
\hline Flavonoids & Radix astragali & $\begin{array}{l}\text { Acquity } \mathrm{UPC}^{2} \mathrm{CSH} \\
\text { Fluoro-Phenyl } \\
\text { (n/a) }\end{array}$ & $\begin{array}{l}\mathrm{A}: \mathrm{CO}_{2}, \mathrm{~B}: \mathrm{MeOH} \\
\text { Gradient elution mode, } 0.5 \mathrm{~mL} / \\
\text { min, } 40^{\circ} \mathrm{C}, 110 \text { bar }\end{array}$ & DAD & $\checkmark$ & [52] \\
\hline Phenolic acids & Wine & $\begin{array}{l}\text { Acquity UPC }{ }^{2} B E H \\
2-E P(3.0 \times 150 \mathrm{~mm}, \\
1.7 \mu \mathrm{m})\end{array}$ & $\begin{array}{l}\mathrm{A}: \mathrm{CO}_{2}, \mathrm{~B}: 0.1 \% \text { TFA in } \mathrm{MeOH} \\
\text { Gradient elution mode, } 2 \mathrm{~mL} / \\
\text { min, } 55^{\circ} \mathrm{C}, 130 \text { bar }\end{array}$ & DAD & $\checkmark$ & [77] \\
\hline Cannabinoids & Cannabis sativa & $\begin{array}{l}\text { Acquity UPC }{ }^{2} \text { BEH } \\
2-E P(3.0 \times 150 \mathrm{~mm} \text {, } \\
1.7 \mu \mathrm{m})\end{array}$ & $\begin{array}{l}\mathrm{A}: \mathrm{CO}_{2}, \mathrm{~B}: 1 \% \text { water in Isopro- } \\
\text { panol/ACN }(8: 2 \mathrm{v} / \mathrm{v}) \\
\text { Gradient elution mode, } 1.4 \mathrm{~mL} / \\
\text { min, } 30^{\circ} \mathrm{C}, 103 \text { bar }\end{array}$ & $\begin{array}{l}\mathrm{DAD} / \mathrm{MS} \\
\text { (ESI) }\end{array}$ & $\checkmark$ & [58] \\
\hline \multicolumn{7}{|l|}{ Miscellaneous } \\
\hline Curcuminoids & Turmeric & $\begin{array}{l}\text { Acquity } \mathrm{UPC}^{2} \mathrm{BEH} \\
(3.0 \times 100 \mathrm{~mm} \\
1.7 \mu \mathrm{m})\end{array}$ & $\begin{array}{l}\text { A: } \mathrm{CO}_{2}, \mathrm{~B}: 10 \mathrm{mmol} \text { oxalic acid } \\
\text { in } \mathrm{MeOH} \\
\text { Gradient elution mode, } 0.9 \mathrm{~mL} / \\
\text { min, } 40^{\circ} \mathrm{C}, 124 \mathrm{bar}\end{array}$ & DAD & - & [78] \\
\hline Destruxins & $\begin{array}{l}\text { Metarhizium } \\
\text { brunneum }\end{array}$ & $\begin{array}{l}\text { Acquity } \mathrm{UPC}^{2} \mathrm{BEH} \\
2-\mathrm{EP}(3.0 \times 100 \mathrm{~mm}, \\
1.7 \mu \mathrm{m})\end{array}$ & $\begin{array}{l}\text { A: } \mathrm{CO}_{2}, \mathrm{~B}: 0.02 \% \text { TFA in } \mathrm{MeOH} / \\
\mathrm{ACN}(8: 2 \mathrm{v} / \mathrm{v}) \\
\text { Gradient elution mode, } 2 \mathrm{~mL} / \\
\text { min, } 60^{\circ} \mathrm{C}, 140 \mathrm{bar}\end{array}$ & $\begin{array}{l}\mathrm{DAD} / \mathrm{MS} \\
\text { (ESI) }\end{array}$ & $\checkmark$ & [63] \\
\hline $\begin{array}{l}\text { Tocopherols and } \\
\text { tocotrienols }\end{array}$ & Soybean oil & $\begin{array}{l}\text { Amine Luna } \mathrm{NH}_{2} \\
(2.0 \times 150 \mathrm{~mm}, \\
3 \mu \mathrm{m})\end{array}$ & $\begin{array}{l}\text { A: } \mathrm{CO}_{2}, \mathrm{~B}: 0.1 \% \text { formic acid in } \\
\text { EtOH } \\
\text { Gradient elution mode, } 1.5 \mathrm{~mL} / \\
\text { min, } 30^{\circ} \mathrm{C}, 130 \text { bar }\end{array}$ & $\begin{array}{l}\mathrm{DAD} / \mathrm{MS} \\
\text { (APPI) }\end{array}$ & $\checkmark$ & $\begin{array}{l}\text { [79] } \\
\text { contin- } \\
\text { ued }\end{array}$ \\
\hline
\end{tabular}


- Table 1 Continued

\begin{tabular}{|c|c|c|c|c|c|c|}
\hline Compounds & Plant species & Stationary phase & Analytical conditions & Detection & Quant. & Ref. \\
\hline $\begin{array}{l}\text { Vitamine } \mathrm{E} \\
\text { isomers }\end{array}$ & $\begin{array}{l}\text { Moringa oleifera } \\
\text { leafs }\end{array}$ & $\begin{array}{l}\text { Acquity } \mathrm{UPC}^{2} \mathrm{BEH} \\
2-\mathrm{EP}(3.0 \times 100 \mathrm{~mm} \text {, } \\
1.7 \mu \mathrm{m})\end{array}$ & $\begin{array}{l}\mathrm{A}: \mathrm{CO}_{2}, \mathrm{~B}: \mathrm{MeOH} / \text { Isopropanol } \\
(1: 1 \mathrm{v} / \mathrm{v}) \\
\text { Gradient elution mode, } 1.5 \mathrm{~mL} / \\
\text { min, } 50^{\circ} \mathrm{C}, 124 \mathrm{bar}\end{array}$ & DAD & $\checkmark$ & [80] \\
\hline $\begin{array}{l}\text { Goitrin and } \\
\text { epigoitrin }\end{array}$ & Isatis indigotica & $\begin{array}{l}(\mathrm{S}, \mathrm{S}) \text {-Whelk-O } 1 \\
(4.6 \times 250 \mathrm{~mm} \\
10 \mu \mathrm{m})\end{array}$ & $\begin{array}{l}\mathrm{A}: \mathrm{CO}_{2}, \mathrm{~B}: \mathrm{MeOH} \\
\text { Gradient elution mode, } 3 \mathrm{~mL} / \\
\text { min, } 40^{\circ} \mathrm{C}, 120 \text { bar }\end{array}$ & $\begin{array}{l}\text { DAD/MS } \\
\text { (APCI) }\end{array}$ & $\checkmark$ & [81] \\
\hline Aflatoxins & Edible oil & $\begin{array}{l}\text { Acquity } \mathrm{UPC}^{2} \mathrm{BEH} \\
2-\mathrm{EP}(2.1 \times 100 \mathrm{~mm} \text {, } \\
1.8 \mu \mathrm{m})\end{array}$ & $\begin{array}{l}\mathrm{A}: \mathrm{CO}_{2}, \mathrm{~B}: \mathrm{MeOH} \\
\text { Gradient elution mode, } 1.0 \mathrm{~mL} / \\
\text { min, } 50^{\circ} \mathrm{C}\end{array}$ & $\begin{array}{l}\text { MS } \\
\text { (ESI) }\end{array}$ & $\checkmark$ & [82] \\
\hline
\end{tabular}

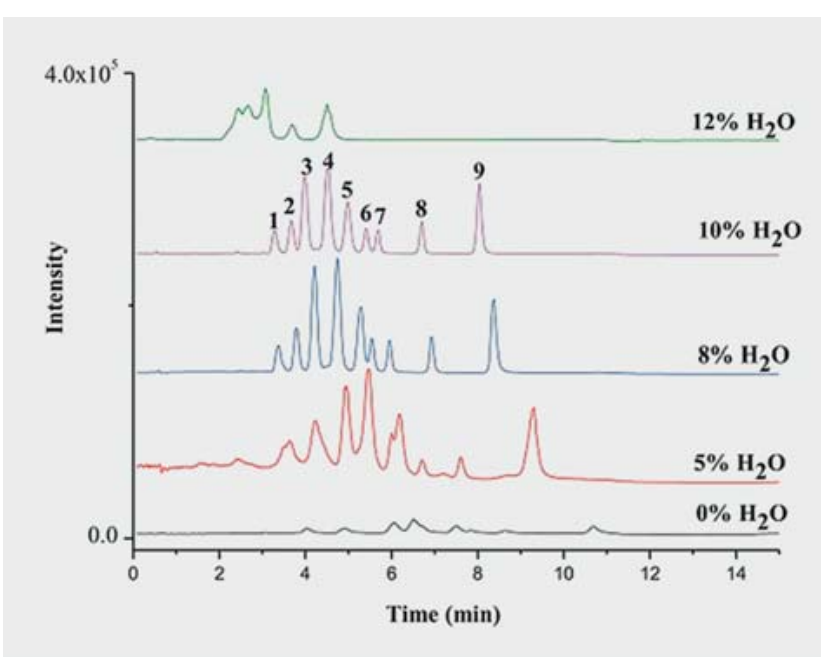

- Fig. 3 Effect of water content in the mobile phase on the separation of nine kudinosides from Ilex latifolia leaves. Peak assignment: 1. Kudinoside F, 2. Kudinoside A, 3. Ilekudinoside G, 4. Kudinoside E, 5. Kudinoside C, 6. Kudinoside G, 7. Latifoloside Q, 8. Latifoloside H, 9. Kudinoside O. Reproduced with permission from [42] [rerif].

the advantage to avoid hydrolysis of ginkgolides that occur during RP-HPLC with aqueous eluents and without the need for derivatization of the ginkgolic acids as it is necessary prior to $G C$ analysis.

Only recently, Zhu et al. [45] compared the separation of 20 different spirostanol saponins by UHPSFC and UHPLC underlining the complementarity of both techniques. While UHPSFC showed to be advantageous for the separation of spirostanol saponins with the same aglycone and a different sugar residue, UHPLC was preferable for the resolution of saponins with the same sugar residue and different aglycones. Up to now, no application to real samples was demonstrated.

\section{Alkaloids}

Few applications focused on analysis of alkaloids in the very early years of SFC; recently reawakened interest in this topic can be observed.
The psychoactive plant Mitragyna specicosa and kratom, a product obtained thereof, are widely used as pain suppressor and low cost substitute for opioids [46]. Due to the addiction potential and toxicity in multiple organ system the U.S. Food and Drug Administration (FDA) has called for detention of all related products [47]. Although mitragynine and 7-hydroxymitragynine are the main psychotropic constituents, other epimeric indole (speciogynine and speciociliatine, which are diastereomeres of mitragynine, paynantheine and 3-isopaynantheine) and oxindole alkaloids (corynoxine A and corynoxine B) are present as well. Wang et al. [46] succeeded in the simultaneous separation of all eight compounds in 7 min using an Agilent RX-SIL column and a mixture of $\mathrm{CO}_{2}$ and methanol containing $10 \mathrm{mmol}$ ammonium acetate as mobile phase. SFC method provided faster separation and superior resolution compared to both UHPLC and GC method. Similar alkaloids were also in the focus of another study: Yang et al. [48] resolved two pairs of 7-epimeric oxindole alkaloids (rhynchophylline and isorhynchophylline, corynoxine A and corynoxine B) from Uncaria macrophylla on a Torus 1-AA column as well as on a Torus Diol column. Acetonitrile (containing $0.1 \%$ DEA or $0.1 \%$ ammonium hydroxide) was chosen as modifier, because of its ability to suppress the epimeric interconversion of these analytes longer than other modifiers. Scaled up to preparative SFC, all four alkaloids were isolated with purities higher than $95 \%$.

Due to the extraordinary toxicity of Aconitum alkaloids, highly sensitive and reliable analytical methods are mandatory for an adequate risk assessment. In a recent publication, separation of five alkaloids in Aconitum pendulum extracts was obtained on an Acquity UPC ${ }^{2}$ BEH 2-EP column [49]. Short runtime (3 min), excellent validation results (recovery rates from 92.3 to $101.2 \%$ ), and LOQ values between 0.03 and $0.08 \mathrm{ng} / \mathrm{mL}$ obtained with an MS detector in the positive ESI mode, indicated that SFC systems can easily keep up with other techniques.

The separation of the amine alkaloids in Piper longum was reached through the offline coupling of SFC and UHPLC [50]. The first dimension was performed on a SFC system using a XAmide column and methanol as modifier. The manually collected, dried fractions were re-dissolved and subsequently analyzed on a UHPLC system and an HSS T3 column (second dimension). Due to the high orthogonality of both systems, not only separation 


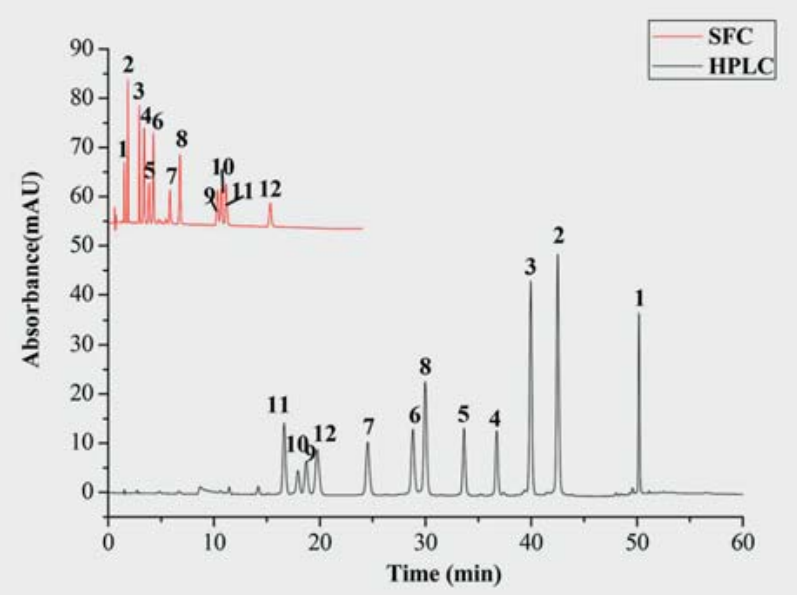

- Fig. 4 Separation of the 12 flavonoids in both SFC and HPLC modes. Peak assignment: 1. Kaempferide, 2. Baicalein, 3. Kaempferol, 4. Luteolin, 5. Quercetin, 6. Morin, 7. Myricetin, 8. Baicalin, 9. Hyperoside, 10. Luteoloside, 11. Myricitrine, 12. Buddleoside. Reproduced with permission from [51] [rerif].

power was increased, but also the detection of low contained compounds, which were overshadowed in the one-dimensional separation, was achieved.

\section{Phenolic compounds}

The frequent occurrence and biological activity render phenolic compounds to one of the most interesting ingredients in plant kingdom. In the last few years an increasing number of analytical applications were investigated focusing on flavonoids [51,52], coumarins [53-55], kavalactones [56], anthraquinones [57], and cannabinoids [58].

Huang et al. [51] presented very recently a first SFC-DAD-UV method for the analysis of 12 flavonoids, among them flavones, flavanols, as well as mono- und diglycosides. Baseline separation was achieved using a ZORBAX RX-SIL column $(4.6 \times 150 \mathrm{~mm}$, $5 \mu \mathrm{m})$ and $0.1 \%$ phosphoric acid in methanol as modifier at a column temperature of $40^{\circ} \mathrm{C}$ and an outlet pressure of 200 bar. The authors compared their SFC method with HPLC analysis of the standard compounds utilizing a ZORBAX SB-C18 column with the same dimensions and particle size. Whereas the SFC analysis took $18 \mathrm{~min}, 55 \mathrm{~min}$ were necessary to obtain baseline separation by HPLC ( Fig. 4). The SFC method was subsequently validated for five representative congeners (limits of quantitation from 2.19 to $5.86 \mu \mathrm{g} / \mathrm{mL}$, recoveries between $100.2 \%$ and $104.1 \%$, precision better than $2.4 \%$ for aglyca and $4.6 \%$ for glycosides) and applied to the quantitative analysis of hydroethanolic extracts of Chrysanthemum marifolium.

A few SFC methods have been published up to now for the determination of coumarins. Pfeifer et al. [53] presented a validated SFC-DAD-UV method for the determination of eight congeners in Angelica dahurica roots, Kim et al. [54] resolved two pyranocumarins in Angelica gigas roots, and Winderl et al. [55] succeeded in the first complete separation of all coumarins in Ammi visnaga fruit.
The first report on the successful separation of anthraquinones has been published as well [57]. Five anthraquinones (chrysophanol, physcion, emodin, aloeemodin, and rhein) could be resolved in less than 5 min on an Acquity UPC ${ }^{2} \mathrm{HSS} \mathrm{C}_{18}$ SB column using methanol with $0.05 \%$ DEA as modifier and DAD detection. The method was validated (LOQs $<1.34 \mu \mathrm{g} / \mathrm{mL}$, recovery rates between $95.4 \%$ and $103.1 \%$, precision better than $6.92 \%$ ) and successfully applied to the analysis of rhubarb extracts.

Cannabis has been used for centuries due to its manifold medicinal properties but was banned as one of the most popular illegal recreational drugs worldwide [59]. Whereas analysis of its constituents was mainly of interest in body fluids and hair samples to prove drug abuse for many years, the situation has changed drastically as marijuana-and not only $\Delta 9-T H C$-has recently been brought into medicinal use in several countries. Due to this changed situation, reliable standardization and quality control is badly needed [60]. Wang et al. [58] presented very recently a promising SFC-DAD/UV-MS method for the quantitative determination of nine of the most abundant cannabinoids. Separation was achieved within $11 \mathrm{~min}$ using isopropanol/acetonitrile $(80: 20 \mathrm{v} / \mathrm{v})$ with $1 \%$ water as modifier and a BEH 2-EP column with sub-2- $\mu \mathrm{m}$ particles. Specificity was proved by MS detection, LOQs were reported as $5 \mu \mathrm{g} / \mathrm{mL}$ for acidic and $10 \mu \mathrm{g} / \mathrm{mL}$ for neutral cannabinoids, recoveries ranged from 96.1 to $107.6 \%$, and the overall precision was better than $7.6 \%$. The method was applied to the analysis of 30 cannabis and hashish samples (acetonitrile/ methanol extracts $[80: 20 \mathrm{v} / \mathrm{v}])$. The results were in good agreement with a standard UHPLC method (variations $\pm 13.0 \%$ ). The SFC-DAD/UV-MS method might be an alternative to existing methods with the advantage of orthogonality to UHPLC, increasing the power of identification of congeners in complex matrices, and without the need for decarboxylation or derivatization as it is necessary prior to GC analysis.

Murauer et al. [56] developed a fast and validated method for the determination of all major lactones in Piper methysticum, a plant that was long considered as an herbal alternative to synthetic anxiolytics but banned from the market due to assumed hepatotoxicity in 2002. Baseline separation was obtained in less than 4 min using an Acquity UPC ${ }^{2} \mathrm{BEH}$ column and a mixture of $\mathrm{CO}_{2}$ and methanol with DEA as mobile phase. With $70^{\circ} \mathrm{C}$ a rather high column temperature, already $10^{\circ} \mathrm{C}$ above the recommended maximum by the column manufacturer was selected, because only under these conditions baseline separation of kavain and yangonin was possible.

Recently, a supercritical based protocol for the extraction, analysis, and isolation of six polar compounds (o-vanillin, styracin, vanillin, trans-cinnamic acid, vanillic acid, and shikimic acid) from Styrax, an exudate from various Liquidambar trees, has been published [61]. A mixture of supercritical $\mathrm{CO}_{2}$ and ethanol $(1: 1)$ was used for the extraction. The generated extracts were resolved on an Acquity UPC ${ }^{2}$ BEH 2-EP column using $0.1 \%$ phosphoric acid in methanol as modifier. Scaled up to preparative SFC, styracin and trans-cinnamic acid were isolated on a Viridis BEH 2-EP column in only $7 \mathrm{~min}$. Compared to conventional workflows, the author described supercritical based methods as a cheap, time-saving, and environmentally friendly alternative that will gain in value in the future. 


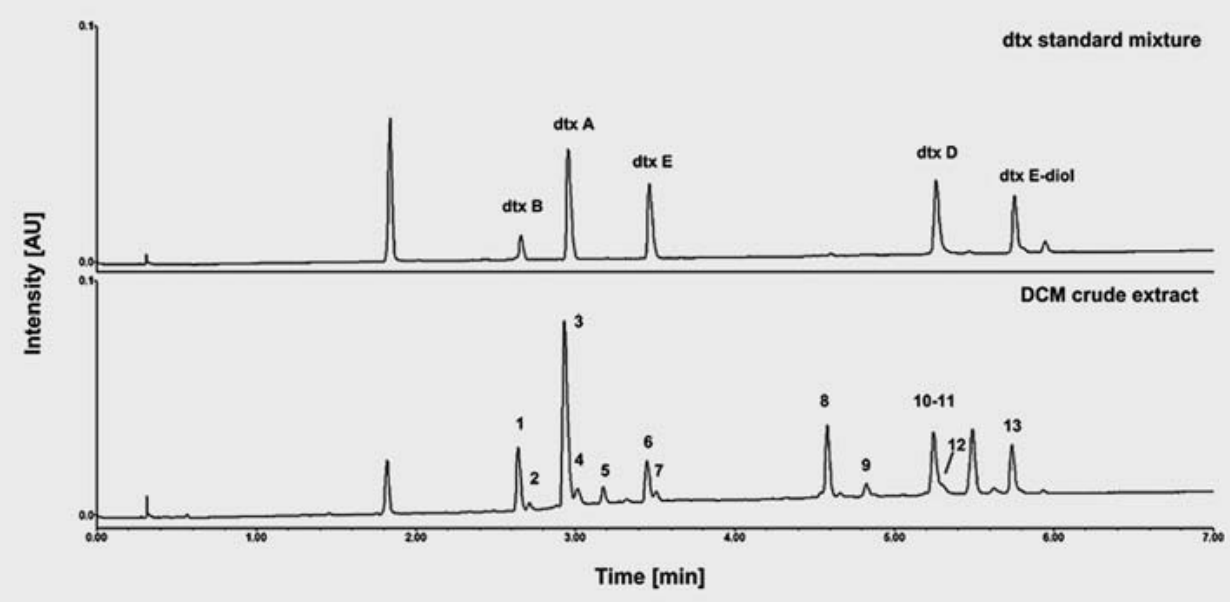

- Fig. 5 Representative UHPSFC-PDA chromatogram of a Metarhizium brunneum DCM crude extract and a destruxine standard mixture containing $\operatorname{dtx} A, B, D, E$, and E-diol. Reproduced with permission from [63].

\section{Miscellaneous}

Cyclic hexadepsipeptides, known as destruxins, are produced by the fungus Metarhizium brunneum, which is used as a pest control agent [62]. Due to concerns that this use entails risks to humans and the environment, the development of validated analysis methods is of great interest. Optimum resolution was obtained on an Acquity UPC ${ }^{2}$ BEH 2-EP column with a mixture of supercritical $\mathrm{CO}_{2}$ and methanol/acetonitrile $(8: 2 \mathrm{v} / \mathrm{v})$ containing $0.02 \%$ trifluoroacetic acid as the mobile phase [63]. As shown in $\mathbf{F i g . 5}$, 17 analytes were separated within 4 min. Five of them were identified using reference material, while the other eight were identified by MS. Compared to established UHPLC method, SFC is characterized by shorter analysis time, rapid equilibration, higher throughput and low operation costs, but has the disadvantage of 4-26 times lower sensitivity. According to the authors, this may be explained by the lower injection volume on one hand and the lower sensitivity of SFC-UV compared to HPLC-UV on the other hand.

Recently, Grand-Guillaume Perrenoud et al. [64] highlighted the versatility of SFC for natural product analysis. A set of 120 highly diverse natural compounds (alkaloids, organic acids, flavonoids, cardioglycosides, etc.) were selected for a systematic column screening on 15 different stationary phases applying identical elution parameters $\left(\mathrm{CO}_{2}\right.$ and $\mathrm{MeOH}$ with $10 \mathrm{mmol}$ ammonium formate and $2 \%$ water). The SFC system was coupled to a Q-ToF mass spectrometer operated in both positive and negative modes with ethanol as make-up liquid. According to their results, the method is suitable for almost $90 \%$ of the tested compounds. Three stationary phases (Diol, not end-capped C18 and 2-EP) showed to be appropriate for wide-range usage. To prove this, dichloromethane and methanol extracts from white willow and yerba mate were analyzed on these three columns under the previously mentioned conditions. The obtained metabolite profiles showed the ability of the developed method for the analysis of both complex polar and nonpolar plant extracts.
Establishing a pharmacokinetic study of a natural product is challenging. Due to the low concentration, complex matrices and wide range of active ingredients, appropriate sensitive and selective analytical methods are indispensable [65]. In comparison to LC-MS, the method of choice for most approaches, SFC is still in its infancy and the number of publications is meagre [66]. Therefore, the following two approaches are noteworthy, as they underline the auspicious potential of SFC in this sector that will surely gain in value in the future.

The four indole alkaloids scholarisine, 19-epischolarisine, vallesamine, and picrinine are described as the major bioactive compounds in Alstonia scholaris, a widely distributed folk medicinal plant in Asia and Africa, used for the treatment of chronic pulmonary diseases. Although commercial formulations (Dengtaiye tablets, DTY) are available, their pharmacokinetic profile is still poorly explored and in vivo studies are missing. Recently, Yang et al. [67] developed a SFC/MS-MS method for the simultaneous quantitation of these four compounds in rat plasma using an Acquity UP$\mathrm{C}^{2} \mathrm{BEH} 2$-EP column with $2 \mathrm{mmol}$ ammonium formate in methanol as modifier. The method was subsequently validated (LOQs $50 \mathrm{pg} / \mathrm{mL}$, recoveries between 84.47 and $95.22 \%$, precision in the range from 1.42 to $12.85 \%$ ) and applied to a pharmacokinetic study in rats after oral administration of $108 \mathrm{mg} / \mathrm{kg}$ Dengtaiye tablets.

The second pharmacokinetic study focused on the simultaneous monitoring of three ginkgolides and their six hydrolyzed metabolites in rat plasma after intravenous administration of the total ginkgolide extract [68]. Methanol with 5\% water and $10 \mathrm{mmol}$ ammonium acetate was used as modifier and a triple quadrupole MS for detection. The use of supercritical $\mathrm{CO}_{2}$ is favorable as it avoids spontaneous hydrolysis of ginkgolides during analysis and allows an accurate characterization of the naturally occurring metabolites. As authentic standards for the ginkgolides metabolites were missing, diazepam and ketoprofen were chosen as internal standards for the method validation (correlation coef- 
ficients $>0.992$, LLOQ between 0.2 and $1.0 \mu \mathrm{g} / \mathrm{mL}$, recoveries of $80.0-116.3 \%$ with RSD less than $10.1 \%$ ).

\section{Future Perspectives and Concluding Remarks}

Whereas in the early days SFC has mainly been restricted to a small group of nonpolar compounds, over the years it has largely expanded its suitability and developed to a versatile technique with great potential for natural product analysis. As shown in the summarized approaches, ranging from nonpolar carotenoids to highly polar glycosides, SFC has finally emerged from the shadow of HPLC and GC, combining some of their best features. Consequently, remarkable short methods are providing high efficient separations at low costs and without the need of toxic solvents. Moreover, the use of supercritical $\mathrm{CO}_{2}$ offers finally an adequate solution for thermolabile and water-unstable plant ingredients inaccessible with HPLC and GC. Often criticized limitations, like poor quantitative performance and limited reliability, could clearly be tackled according to recent publications presenting separations with low LOQ values and excellent validation criteria. The upscale from analytical to preparative SFC provides a cost-saving and rapid solution for the isolation of highly pure substances due to easy solvent removal and the possibility of high sample loading. With all these features and the continuously ongoing technical development, SFC has become a promising analytical tool with a bright future in the area of natural product analysis.

\section{Conflict of Interest}

The authors declare no conflicts of interest.

\section{References}

[1] Krüger $\mathrm{H}$, Schulz $\mathrm{H}$. Analytical techniques for medicinal and aromatic plants. Stewart Post Harvest Rev 2007; 3: 1-12

[2] Desfontaine V, Guillarme D, Francotte E, Nováková L. Supercritical fluid chromatography in pharmaceutical analysis. J Pharm Biomed Anal 2015; 113: $56-71$

[3] Donato P, Inferrera V, Sciarrone D, Mondello L. Supercritical fluid chromatography for lipid analysis in foodstuffs. J Sep Sci 2017; 40: 361-382

[4] Wang Y. Chapter 11 - Supercritical fluid chromatography of natural products. In: Webster GK, ed. Supercritical Fluid Chromatography: Advances and Applications in Pharmaceutical Analysis. Singapore: Pan Stanford Publ; 2014: 297-331

[5] Berger TA. Separation of polar solutes by packed column supercritical fluid chromatography. J Chromatogr A 1997; 785: 3-33

[6] Lesellier $E$, West $C$. The many faces of packed column supercritical fluid chromatography - a critical review. J Chromatogr A 2015; 1382: 2-46

[7] Hartmann A, Ganzera M. Supercritical fluid chromatography - theoretical background and applications on natural products. Planta Med 2015; 81: 1570-1581

[8] Ganzera M, Murauer A. Chapter 15 - separation of natural products. In: Poole CF, ed. Supercritical Fluid Chromatography. Amsterdam: Elsevier; 2017: 439-460

[9] Saito M. History of supercritical fluid chromatography. J Biosci Bioeng 2013; 115: 590-599
[10] Guiochon G, Tarafder A. Fundamental challenges and opportunities for preparative supercritical fluid chromatography. J Chromatogr A 2011; 1218: 1037-1114

[11] De Klerck K, Mangelings D, Vander Heyden Y. Supercritical fluid chromatography for the enantioseparation of pharmaceuticals. J Pharm Biomed Anal 2012; 69: 7-92

[12] Farrell WP, Aurigemma CM, Masters-Moore DF. Advances in high throughput supercritical fluid chromatography. J Liq Chromatogr Relat Techno 2009; 32: 1689-1710

[13] Berger TA. Instrumentation for analytical scale supercritical fluid chromatography. J Chromatogr A 2015; 1421: 171-183

[14] Lesellier E. Retention mechanisms in super/subcritical fluid chromatography on packed columns. J Chromatogr A 2009; 1216: 1881-1890

[15] Bamba T. Application of supercritical fluid chromatography to the analysis of hydrophobic metabolites. J Sep Sci 2008; 31: 1274-1278

[16] Wang RQ, Ong TT, Ng SQ, Tang W. Recent advances in pharmaceutical separations with supercritical fluid chromatography using chiral stationary phases. Trends Analyt Chem 2012; 37: 83-100

[17] Chester TL, Pinkston JD. Supercritical Fluid and Unified Chromatography Anal Chem 2004; 76: 4606-4613

[18] Nováková L, Grand-Guillaume Perrenoud A, Francois I, West C, Lesellier E, Guillarme D. Modern analytical supercritical fluid chromatography using columns packed with sub- $2 \mu \mathrm{m}$ particles: a tutorial. Anal Chim Acta 2014; 824: 18-35

[19] Grand-Guillaume Perrenoud A, Veuthey JL, Guillarme D. The use of columns packed with sub- $2 \mu \mathrm{m}$ particles in supercritical fluid chromatography. Trends Analyt Chem 2014; 63: 44-54

[20] Poole CF. Stationary phases for packed-column supercritical fluid chromatography. J Chromatogr A 2012; 1250: 157-171

[21] Lesellier E. Overview of the retention in subcritical fluid chromatography with varied polarity stationary phases. J Sep Sci 2008; 31: 1238-1251

[22] West C, Lemasson E, Bertin S, Hennig P, Lesellier E. An improved classification of stationary phases for ultra-high performance supercritical fluid chromatography. J Chromatogr A 2016; 1440: 212-228

[23] Galea C, Mangelings D, Vander Heyden Y. Characterization and classification of stationary phases in HPLC and SFC - a review. Anal Chim Acta 2015; 886: 1-15

[24] Ashraf-Khorassani M, Taylor LT, Seest E. Screening strategies for achira supercritical fluid chromatography employing hydrophilic interaction liquid chromatography-like parameters. J Chromatogr A 2012; 1229: 237-248

[25] Berger TA, Berger BK. Minimizing UV noise in supercritical fluid chromatography. I. Improving back pressure regulator pressure noise. J Chromatogr A 2011; 1218: 2320-2326

[26] Desfontaine V, Veuthey JL, Guillarme D. Chapter 8 - Hyphenated detectors: mass spectrometry. In: Poole CF, ed. Supercritical Fluid Chromatography. Amsterdam: Elsevier; 2017: 213-238

[27] Suzuki M, Nishiumi S, Kobayashi T, Sakai A, Iwata Y, Uchikata T, Izumi Y, Azuma T, Bamba T, Yoshida M. Use of on-line supercritical fluid extraction-supercritical fluid chromatography/tandem mass spectrometry to analyze disease biomarkers in dried serum spots compared with serum analysis using liquid chromatography/tandem mass spectrometry: SFE-SFC/MS/MS to analyze disease biomarkers in dried serum spots. Rapid Commun Mass Spectrom 2017; 31: 886-894

[28] Schad G. SFE-SFC-MS for the analysis of pesticide residues in food products. The Column 2015; 11

[29] Taylor LT. Supercritical fluid chromatography for the 21st century. J Supercrit Fluids 2009; 47: 566-573

[30] Kalikova K, Slechtova T, Vozka J, Tesarova E. Supercritical fluid chromatography as a tool for enantioselective separation; a review. Anal Chim Acta 2014; 821: 1-33 
[31] Webster GK. Supercritical Fluid Chromatography: Advances and Applications in Pharmaceutical Analysis. Singapore: Pan Stanford Publ; 2014

[32] Poole CF. Supercritical Fluid Chromatography: Handbooks in Separation Science. Amsterdam: Elsevier; 2017

[33] Bamba T, Lee JW, Matsubara A, Fukusaki E. Metabolic profiling of lipids by supercritical fluid chromatography/mass spectrometry. J Chromatogr A 2012; 1250: 212-219

[34] Yamada T, Taguchi, K., Bamba T. Chapter 14 - separation of lipids. In: Poole CF, ed. Supercritical Fluid Chromatography. Amsterdam: Elsevier; 2017: 419-438

[35] Laboureur L, Ollero M, Touboul D. Lipidomics by supercritical fluid chromatography. Int J Mol Sci 2015; 16: 13868-13884

[36] Bernal JL, Martin MT, Toribio L. Supercritical fluid chromatography in food analysis. J Chromatogr A 2013; 1313: 24-36

[37] Lísa M, Holčapek M. High-throughput and comprehensive lipidomic analysis using ultrahigh-performance supercritical fluid chromatography - mass spectrometry. Anal Chem 2015; 87: 7187-7195

[38] McLaren L, Myers MN, Giddings JC. Dense-gas chromatography of nonvolatile substances of high molecular weight. Science 1968; 159: 197199

[39] Bonaccorsi I, Cacciola F, Utczas M, Inferrera V, Giuffrida D, Donato P, Dugo P, Mondello L. Characterization of the pigment fraction in sweet bell peppers (Capsicum annuum L.) harvested at green and overripe yellow and red stages by offline multidimensional convergence chromatography/liquid chromatography - mass spectrometry. J Sep Sci 2016; 39: 3281-3291

[40] Rodriguez-Amaya DB. Food Carotenoids: Chemistry, Biology and Technology. Chichester: Wiley-Blackwell; 2015

[41] Giuffrida D, Zoccali M, Giofrè SV, Dugo P, Mondello L. Apocarotenoids determination in Capsicum chinense Jacq. cv. Habanero, by supercritical fluid chromatography-triple-quadrupole/mass spectrometry. Food Chem 2017; 231: 316-323

[42] Huang Y, Zhang T, Zhou H, Feng Y, Fan C, Chen W, Crommen J, Jiang Z. Fast separation of triterpenoid saponins using supercritical fluid chromatography coupled with single quadrupole mass spectrometry. J Pharm Biomed Anal 2016; 121: 22-29

[43] Thompson J, Strode B, Taylor LT, van Beek TA. Supercritical fluid chromatography of ginkgolides A, B, C and J and bilobalide. J Chromatogr A 1996; 738: 115-122

[44] Wang M, Carrell E], Chittiboyina AG, Avula B, Wang YH, Zhao J, Parcher JF, Khan IA. Concurrent supercritical fluid chromatographic analysis of terpene lactones and ginkgolic acids in Ginkgo biloba extracts and dietary supplements. Anal Bioanal Chem 2016; 408: 4649-4660

[45] Zhu LL, Zhao Y, Xu YW, Sun QL, Sun XG, Kang LP, Yan RY, Zhang J, Liu C, Ma BP. Comparison of ultra-high performance supercritical fluid chromatography and ultra-high performance liquid chromatography for the separation of spirostanol saponins. J Pharm Biomed Anal 2016; 120: 7278

[46] Wang M, Carrell EJ, Ali Z, Avula B, Avonto C, Parcher JF, Khan IA. Comparison of three chromatographic techniques for the detection of mitragynine and other indole and oxindole alkaloids in Mitragyna speciosa (kratom) plants: liquid chromatography. J Sep Sci 2014; 37: 1411-1418

[47] FDA Import Alert 54-15. Detention without physical examination of dietary supplements and bulk dietary ingredients that are or contain Mitragyna speciosa or kratom. Published on November 7, 2015. Available from https://www.accessdata.fda.gov/cms_ia/importalert_1137.html; Accessed August 20, 2017

[48] Yang W, Zhang Y, Pan H, Yao C, Hou J, Yao S, Cai L, Feng R, Wu W, Guo D. Supercritical fluid chromatography for separation and preparation of tautomeric 7-epimeric spiro oxindole alkaloids from Uncaria macrophylla. J Pharm Biomed Anal 2017; 134: 352-360

[49] Zhao T], Qi HY, Chen J, Shi YP. Quantitative analysis of five toxic alkaloids in Aconitum pendulum using ultra-performance convergence chroma- tography (UPC ${ }^{2}$ ) coupled with mass spectrometry. RSC Adv 2015; 5 : 103869-103875

[50] Li K, Fu Q, Xin H, Ke Y, Jin Y, Liang X. Alkaloids analysis using off-line twodimensional supercritical fluid chromatography $\times$ ultra-high performance liquid chromatography. Analyst 2014; 139: 3577-3587

[51] Huang Y, Feng Y, Tang G, Li M, Zhang T, Fillet M, Crommen J, Jiang Z. Development and validation of a fast SFC method for the analysis of flavonoids in plant extracts. J Pharm Biomed Anal 2017; 140: 384-391

[52] Wang B, Liu X-H, Zhou W, Hong Y, Feng SL. Fast separation of flavonoids by supercritical fluid chromatography using a column packed with a sub$2 \mu \mathrm{m}$ particle stationary phase. J Sep Sci 2017; 40: 1410-1420

[53] Pfeifer I, Murauer A, Ganzera M. Determination of coumarins in the roots of Angelica dahurica by supercritical fluid chromatography. J Pharm Biomed Anal 2016; 129: 246-251

[54] Kim HS, Chun JM, Kwon BI, Lee AR, Kim HK, Lee AY. Development and validation of an ultra-performance convergence chromatography method for the quality control of Angelica gigas Nakai: other techniques. J Sep Sci 2016; 39: 4035-4041

[55] Winderl B, Schwaiger S, Ganzera M. Fast and improved separation of major coumarins in Ammi visnaga (L.) Lam. by supercritical fluid chromatography. J Sep Sci 2016; 39: 4042-4048

[56] Murauer A, Ganzera M. Quantitative determination of lactones in Piper methysticum (kava-kava) by supercritical fluid chromatography. Planta Med 2017; 83: 1053-1057

[57] Aichner D, Ganzera M. Analysis of anthraquinones in rhubarb (Rheum palmatum and Rheum officinale) by supercritical fluid chromatography. Talanta 2015; 144: 1239-1244

[58] Wang M, Wang YH, Avula B, Radwan MM, Wanas AS, Mehmedic Z, van Antwerp J, ElSohly MA, Khan IA. Quantitative determination of cannabinoids in cannabis and cannabis products using ultra-high-performance supercritical fluid chromatography and diode array/mass spectrometric detection. J Forensic Sci 2017; 62: 602-611

[59] National Academies of Sciences, Engineering, and Medicine. The Health Effects of Cannabis and Cannabinoids: The Current State of Evidence and Recommendations for Research. Washington, DC: The National Academies Press; 2017: 1-3

[60] Thomas BF, ElSohly MA. The analytical chemistry of cannabis: quality assessment, assurance, and regulation of medicinal marijuana and cannabinoid preparations. Amsterdam: Elsevier/RTI International; 2016

[61] Scheuba J, Wronski VK, Rollinger J, Grienke U. Fast and green - CO2 based extraction, isolation, and quantification of phenolic styrax constituents. Planta Med 2017; 83: 1068-1075

[62] Pedras MSC, Irina Zaharia L, Ward DE. The destruxins: synthesis, biosynthesis, biotransformation, and biological activity. Phytochemistry 2002; 59: 579-596

[63] Taibon J, Sturm S, Seger C, Werth M, Strasser H, Stuppner H. Supercritical fluid chromatography as an alternative tool for the qualitative and quantitative analysis of Metarhizium brunneum metabolites from culture broth. Planta Med 2015; 81: 1736-1743

[64] Grand-Guillaume Perrenoud A, Guillarme D, Boccard J, Veuthey JL, Barron D, Moco S. Ultra-high performance supercritical fluid chromatography coupled with quadrupole-time-of-flight mass spectrometry as a performing tool for bioactive analysis. J Chromatogr A 2016; 1450: 101-111

[65] Bhattaram VA, Graefe U, Kohlert C, Veit M, Derendorf H. Pharmacokinetics and bioavailability of herbal medicinal products. Phytomedicine Int J Phytother Phytopharm 2002; 9 Suppl 3: 1-33

[66] Desfontaine V, Nováková L, Guillarme D. SFC-MS versus RPLC-MS for drug analysis in biological samples. Bioanalysis 2015; 7: 1193-1195

[67] Yang Z, Sun L, Liang C, Xu Y, Cao J, Yang Y, Gu J. Simultaneous quantitation of the diastereoisomers of scholarisine and 19-epischolarisine, vallesamine, and picrinine in rat plasma by supercritical fluid chromatography with tandem mass spectrometry and its application to a pharmacokinetic study: other techniques. J Sep Sci 2016; 39: 2652-2660 
[68] Liu XG, Qi LW, Fan ZY, Dong X, Guo RZ, Lou FC, Fanali S, Li P, Yang H. Accurate analysis of ginkgolides and their hydrolyzed metabolites by analytical supercritical fluid chromatography hybrid tandem mass spectrometry. J Chromatogr A 2015; 1388: 251-258

[69] Jumaah F, Plaza M, Abrahamsson V, Turner C, Sandahl M. A fast and sensitive method for the separation of carotenoids using ultra-high performance supercritical fluid chromatography-mass spectrometry. Anal Bioanal Chem 2016; 408: 5883-5894

[70] Li B, Zhao H, Liu J, Liu W, Fan S, Wu G, Zhao R. Application of ultra-high performance supercritical fluid chromatography for the determination of carotenoids in dietary supplements. J Chromatogr A 2015; 1425: 287-292

[71] Huang Y, Zhang T, Zhao Y, Zhou H, Tang G, Fillet M, Crommen J, Jiang Z. Simultaneous analysis of nucleobases, nucleosides and ginsenosides in ginseng extracts using supercritical fluid chromatography coupled with single quadrupole mass spectrometry. J Pharm Biomed Anal 2017; 144: 213-219

[72] Kim HS, Moon BC, Choi G, Kim WJ, Lee AY. Ultra-performance convergence chromatography for the quantitative determination of bioactive compounds in Aralia continentalis Kitagawa as quality control markers. J Sep Sci 2017; 40: 2071-2079

[73] Jones MD, Avula B, Wang YH, Lu L, Zhao J, Avonto C, Isaac G, Meeker L, Yu K, Legido-Quigley C, Smith N, Khan IA. Investigating sub-2 $\mu \mathrm{m}$ particle stationary phase supercritical fluid chromatography coupled to mass spectrometry for chemical profiling of chamomile extracts. Anal Chim Acta 2014; 847: 61-72

[74] Végh K, Riethmüller E, Tóth A, Alberti Á, Béni S, Balla J, Kéry Á. Convergence chromatographic determination of camphor in the essential oil of Tanacetum parthenium L.: Convergence chromatographic determination of camphor in essential oil. Biomed Chromatogr 2016; 30: 2031-2037
[75] Fu Q, Li Z, Sun C, Xin H, Ke Y, jin Y, Liang X. Rapid and simultaneous analysis of sesquiterpene pyridine alkaloids from Tripterygium wilfordii Hook. f. Using supercritical fluid chromatography-diode array detector-tandem mass spectrometry. J Supercrit Fluids 2015; 104: 85-93

[76] Li Z, Fu Q, Li K, Liang T, Jin Y. Fast analysis of indole alkaloids from Evodiae fructus by supercritical fluid chromatography. Se Pu Chin J Chromatogr 2014; 32: 506-512

[77] Ovchinnikov DV, Kosyakov DS, Ul'yanovskii NV, Bogolitsyn KG, Falev DI, Pokrovskiy OI. Determination of natural aromatic acids using supercritical fluid chromatography. Russ J Phys Chem B 2016; 10: 1062-1071

[78] Song W, Qiao X, Liang W, Ji S, Yang L, Wang Y, Xu YW, Yang Y, Guo D, Ye M. Efficient separation of curcumin, demethoxycurcumin, and bisdemethoxycurcumin from turmeric using supercritical fluid chromatography. J Sep Sci 2015; 38: 3450-3453

[79] Méjean M, Brunelle A, Touboul D. Quantification of tocopherols and tocotrienols in soybean oil by supercritical-fluid chromatography coupled to high-resolution mass spectrometry. Anal Bioanal Chem 2015; 407 : 5133-5142

[80] Qi N, Gong X, Feng C, Wang X, Xu Y, Lin L. Simultaneous analysis of eight vitamin E isomers in Moringa oleifera Lam. leaves by ultra performance convergence chromatography. Food Chem 2016; 207: 157-161

[81] Wang R, Runco J, Yang L, Yu K, Li Y, Chen R, Wang Z. Qualitative and quantitative analyses of goitrin-epigoitrin in Isatis indigotica using supercritical fluid chromatography-photodiode array detector-mass spectrometry. RSC Adv 2014; 4: 49257-49263

[82] Lei F, Li C, Zhou S, Wang D, Zhao Y, Wu Y. Hyphenation of supercritical fluid chromatography with tandem mass spectrometry for fast determination of four aflatoxins in edible oil: hyphenation of supercritical fluid chromatography with tandem mass spectrometry. Rapid Commun Mass Spectrom 2016; 30: 122-127 